${ }^{16}$ C. C. J. RoothaAN, "A study of two-center integrals useful in calculations on molecular structure II," Jn. Chem. Phys., v. 19, 1951, p. 1445-1458.

${ }^{17} \mathrm{~K}$. RÜDENBERG-reference (2).

${ }_{18}$ Quantum-mechanical methods in valence theory, Sept. 1951, Nat. Acad. Sci. and Office of Naval Research, New York.

\title{
Characters of the Symmetric Groups of Degree 15 and 16
}

Introduction. The construction of the complete character table for the symmetric group of a given degree $n$ (i.e., the group formed by the permutations of $n$ quantities) is a problem of long standing. Theoretically the problem was solved many years ago by FroBENIUS, ${ }^{1}$ who first in troduced the concept of the character of a representation (thus meriting the title of founder of the theory of group representations). Frobenius' expression for the symmetric group characters as coefficients of a certain algebraic form is, however, unsuitable for practical calculation except for groups of quite low degree. Some years ago MURnaGHAN ${ }^{2}$ was able to derive a recursion scheme which permits one to calculate the characters for a given $n$ in terms of the (presumably known) characters of all the symmetric groups of lower degree. This scheme has been employed by various authors ${ }^{3}$ to construct character tables for all the symmetric groups of degree $n \leqslant 14$. The recursion scheme effectively reduces the problem to an exercise in bookkeeping, but, despite its simplicity, it suffers from two practical faults. First of all, the labor involved in computing the characters by hand becomes prohibitive for $n$ larger than, say, 14. Secondly, as mentioned above, for each $n$ the results for all lower $n$ must be used, so that errors may be expected to propagate. This, of course, is not a theoretical limitation, since in principle each table may be calculated, $a b$ initio, and any given table can be checked for consistency by using the fact that the characters themselves satisfy certain orthogonality relations. The impracticability of proceeding by hand computation is clear when it is remembered that the number of characters of the symmetric group of degree $n$ is equal to the square of the number of unrestricted partitions of $n$; for $n=15$, this number is 30,976 .

It should be evident that the practical difficulties mentioned in the preceding paragraph can in large measure be overcome by the use of electronic computers. In fact, it seemed to us that this very calculation would provide a very good test of the speed and flexibility of such a computer with regard to the handling of purely algebraic problems. Accordingly, we set up and carried out the calculation of the complete character tables of the symmetric groups of degree $n=15$ and 16 using the Los Alamos Electronic Computer (MANIAC). The method was checked by re-computing the tables ${ }^{4}$ for $n=10$ to $n=14$. The method of calculation is described in Sec. (III). The experience was quite encouraging, and suggests that it would be profitable to apply electronic computer techniques to a large class of quite complicated problems in algebra and group theory.

The Character Formulae. For convenience we give here a brief resume of the relevant properties of the symmetric group characters. For proofs and detailed discussion the reader is referred to the standard works of LitTLEWOOD $^{5}$ and MuRNAGHAN. ${ }^{6}$ 
The basic fact concerning the symmetric group of degree $n$ is that both its classes and its irreducible representations are in one-to-one correspondence with the number of unrestricted partitions of the integer $n$. More precisely, each class may be denoted by a partition of $n$ into non-negative integers:

$$
\begin{aligned}
& (\rho) \equiv\left(\rho_{1}, \rho_{2} \cdots \rho_{k}\right) \\
& \rho_{1} \geqslant \rho_{2} \geqslant \cdots \geqslant \rho_{k} \\
& \sum_{i=1}^{k} \rho_{i}=n
\end{aligned}
$$

Similarly, each irreducible representation may be so labelled. In the sequel we shall reserve the symbol $(\rho)$ to label classes, and the symbols $(\lambda)$, $(\mu)$ to label irreducible representations. In the interest of compactness we adopt the standard convention of denoting repeated parts by a super-script; thus the partition $(4,3,3,3,1,1)$ of 15 will be written $\left(4,3^{3}, 1^{2}\right)$. With regard to the labelling of classes, this notation has a simple interpretation in terms of the one-dimensional permutation representation of the group, the class $\left(4,3^{3}, 1^{2}\right)$ being that which consists of one cycle of order 4 , three cycles of order 3 , and two cycles of order 1 . For a given irreducible representation $(\lambda)$, the character of the class $(\rho)$ (i.e., the common trace of the matrices belonging to this class and representation) will be denoted by $\chi_{(\rho)}^{(\lambda)}$. For the symmetric group, $\chi$ is always an integer, positive, negative, or zero. If we consider the partition $(\rho)$ as labelling the rows and $(\lambda)$ as labelling the columns, the quantities $\chi_{(\rho)}^{(\lambda)}$ are the elements of a matrix of degree equal to the number of partitions of $n$. The $\chi_{(\rho)}^{(\lambda)}$ satisfy the following orthogonality relations:

$$
\begin{aligned}
& \sum_{(\rho)} h_{\rho} \chi_{(\rho)}^{(\lambda)} \chi_{(\rho)}^{(\mu)}=n ! \delta_{\lambda \mu} \\
& \sum_{(\lambda)} \chi_{(\rho)}^{(\lambda)} \chi_{(\rho)}^{(\lambda)}=\frac{n !}{h_{\rho}} \delta_{\rho \rho^{\prime}}
\end{aligned}
$$

where $h_{\rho}$ is the number of group elements belonging to the class $(\rho)$ and is given explicitly by

$$
h_{\rho}=\frac{n !}{1^{\alpha} \alpha ! 2^{\beta} \beta ! 3^{\gamma} \gamma ! \cdots}
$$

$\alpha$ being the number of unary cycles in $(\rho), \beta$ the number of binary cycles, etc.

For every class, the character of the irreducible representation $(n)$ (corresponding to the partition of $n$ into one part) is unity; i.e.,

$$
\chi_{(\rho)}^{(n)}=1 \text { for all }(\rho)
$$

Similarly, for every class, the character corresponding to the partition $\left(1^{n}\right)$ is \pm 1 , depending on whether the class $(\rho)$ is even (i.e., has an even number of cycles of even order) or odd.

$$
\begin{array}{ll}
\chi_{(\rho)}^{(1 n)}=+1, & (\rho) \text { even } \\
\chi_{(\rho)}^{(1 n)}=-1, & (\rho) \text { odd }
\end{array}
$$

If we denote by $(\tilde{\lambda})$ the partition of $n$ conjugate $^{7}$ to $(\lambda)$, then for every 
class, the characters of the irreducible representations corresponding to $(\tilde{\lambda})$ and $(\lambda)$ are related by:

$$
\chi_{(\rho)}^{\tilde{(})}=\chi_{(\rho)}^{(1 n)} \cdot \chi_{(\rho)}^{(\lambda)}
$$

The consequence of this last relation is that for each $(\rho)$ we have to calculate only slightly more than half the characters, since the character of the associated representation is either equal to, or just the negative of, the character of the given representation. If $P(n)$ denotes the total number of unrestricted partitions of $n$ and $P_{s}(n)$ the number of self-conjugate partitions, then the total number ${ }^{8}$ of quantities $\chi_{(\rho)}^{(\lambda)}$ which must be calculated is just $\frac{1}{2} P(n)\left[P(n)+P_{s}(n)\right]$. For $n=15$ this number is 15,840 .

The recurrence relations for the characters referred to in the introduction may be stated in the following form $:^{5}$

Let $\left(\rho^{\prime}\right)$ be a partition of the integer $(n-m)$ which differs from the partition ( $\rho$ ) of $n$ by the omission of a cycle of order $m$. Then

$$
\chi_{(\rho)}^{(\lambda)}=\sum_{(\mu)} \pm \chi_{(\rho)}^{(\mu)}
$$

where the partitions $(\mu)$ of $(n-m)$ run over the set

$$
(\mu)=\left(\lambda_{1}-m, \lambda_{2}, \cdots \lambda_{k}\right),\left(\lambda_{1}, \lambda_{2}-m, \lambda_{3} \cdots \lambda_{k}\right), \cdots,\left(\lambda_{1}, \lambda_{2} \cdots \lambda_{k}-m\right)
$$

The partitions (8) do not in general satisfy the conditions (1), since the parts will not necessarily be in non-ascending order or even positive. It can be shown ${ }^{5,6}$ that the following three rules may be invoked:

1. If $(\mu)$ has all its parts in non-ascending order but the last part is negative, then the contribution $\chi^{(\mu)}$ to the sum (7) is identically zero.

2. If the $i$ th part of $(\mu)$ is one less than the $i+1$ st part, $\mu_{i}+1=\mu_{i+1}$, then the corresponding contribution to (7) is identically zero.

3. $\chi^{\left(\mu^{\prime}\right)}=-\chi^{\left(\mu^{\prime \prime}\right)}$ where $\left(\mu^{\prime \prime}\right)$ is the partition obtained from $\left(\mu^{\prime}\right)$ by the replacements

$$
\begin{aligned}
& \mu_{i}^{\prime}+1 \rightarrow \mu_{i+1}^{\prime \prime} \\
& \mu_{i+1}^{\prime}-1 \rightarrow \mu_{i}^{\prime \prime}
\end{aligned}
$$

The procedure is thus to operate on the set (8) with rule 3 until either a partition satisfying (1) is obtained or the conditions of rule 1 or 2 are satisfied. (The \pm sign in (7) is intended to represent possible sign changes arising from rule 3.) In conjunction with these operating rules, (7) effectively reduces the calculation of the characters of a given group to those of groups of lower degree. By iteration of this process a complete reduction can be carried out for every given $(\rho)$ until $(\rho)=(0)$. Of course no useful closed expression for this process exists. Formulae do exist ${ }^{5,6}$ which give $\chi_{(\rho)}^{(\lambda)}$ directly in terms of the number of cycles of various orders occurring in $(\rho)$, but they become prohibitively complicated unless $(\lambda)$ has a particularly simple form. There is, however, one explicit formula, due to Frobenius, which is of use in a practical calculation, namely that which expresses the character of the identity class $(p)=\left(1^{n}\right)$ in terms of the parts of $(\lambda)$. This may be written in the form ${ }^{6}$

$$
\chi_{(1 n)}^{(\lambda)}=\frac{n ! \prod_{i<j}\left(l_{i}-l_{j}\right)}{l_{1} ! l_{2} ! \cdots l_{k} !} \quad\left(\lambda=\lambda_{1}, \lambda_{2}, \cdots, \lambda_{k}\right)
$$


where $l_{i}=\lambda_{i}+k-i$. This gives the trace of the unit matrix in the representation $(\lambda)$ and is hence the dimension of the representation. ${ }^{9}$

This completes our resume of the properties of the characters of the symmetric group. Many other relations exist, but they are not of interest for our purpose.

Calculation Scheme. The computer first simply prepares a table of the partitions of $n$. This table is also used to designate the classes. In addition the complete character tables for the symmetric groups of degree $n \leq 4$ are stored in the computer. Their function will be described presently

The sequence of steps performed by the computer is as follows: a character is first decomposed by (7) into a sum of characters all of the same class $\left(\rho^{\prime}\right)$, corresponding to a group of degree $n^{\prime}(<n)$. Attention is fixed on the first component. The integers describing the partition are rearranged, if necessary, in non-ascending order, with the aid of (9). Next the partition is examined to see if

(i) its value is zero (cf. rules 1 and 2, page 214). If it is so, we proceed to the next component of the decomposition; if not, we test to see if

(ii) $n^{\prime} \leq 4$. If this condition is satisfied, the computer makes reference to the appropriate character table stored in the memory; if this condition is not satisfied, the computer finally determines if

(iii) $\left(\rho^{\prime}\right)=\left(1^{n^{\prime}}\right)$. If so, the character is computed by $(10)$. If not, a further reduction by means of $(7)$ is made and the above procedures repeated until the first component is evaluated; all members of the original decomposition are treated in turn.

Obviously, the most efficient method of utilizing (7) is to choose the reduction parameter $m$ as large as possible, and this was always done.

The instructions, or code, for the problem occupied about one-half of the 1024 locations of the electrostatic memory; numerical storage accounted for the remaining half; the partitions occupied a small fraction of the auxiliary drum memory. The computing time for the group $n=15$ was five hours; for $n=16$, twelve hours were necessary to compute the 27,258 distinct characters.

Obviously, the number of operations necessary to compute a character is very heavily dependent upon the structure of the partition $(\rho)$. Indeed, for the class $(\rho)=\left(2^{8}\right)$ six levels of branching were required to obtain the necessary reduction and the computer took thirty-seven minutes to calculate the characters for this class. In comparison only one minute was needed to compute the characters for $(\rho)=(16)$.

Although electrostatic storage limitations precluded a complete orthogonality check on the table, (2b) was used for each class separately.

The two tables of characters have been photographed on microfilm directly from the computer print-out. Each frame contains the characters of one class. These films are available in the UMT FILE [UMT 195].

Robert L. Bivins

N. Metropolis

Paul R. Stein

Los Alamos Scientific Lab.

Mark B. Wells

Univ. of Calif.

Los Alamos, New Mexico. 
${ }^{1}$ G. Frobenius, Preuss. Akad., Berlin, SitzBer., 1900, p. 516. A list of Frobenius' relevant papers may be found in ref. 4 or 5 .

2 F. J. Murnaghan, Am. Journal of Math., v. 59, 1937, p. 437. See also the paper of LITTLEWOOD \& RICHARDSON. ${ }^{3}$

${ }^{3}$ D. E. LitTlewood \& A. R. Richardson, Roy. Soc., Phil. Trans., v. 233A, 1934, p. 99. p. 340 .

4 M. ZiA-ud-Din, London Math. Soc. Proc (2), v. 39, 1935, p. 200; ibid., v. 42, 1937,

K. Kondo, Phys. Math. Soc. Japan, v. 22, 1940, p. 585.

5 D. E. Litrlewood: The Theory of Group Characters, 2nd edition. Oxford, 1950.

- F. J. MuRnaGHan: The Theory of Group Representations. Baltimore, 1938.

7 The partition conjugate to $(\lambda)$ is obtained by interchanging rows and columns in the Ferrers-Sylvester graph of $(\lambda)$.

${ }_{8} P_{8}(n)=P(n)-2 P(n-2)+2 P(n-8)-2 P(n-18)+\cdots$. This was pointed out to us in a private communication from Professor N. J. FINE.

9 The following question is of some practical interest: given $n$, for what partition $(\lambda)$ does (9) take its largest value, and how does this value vary with $n$ ? The authors have been unable to find any discussion of this problem in the literature.

\section{RECENT MATHEMATICAL TABLES}

1234[A].-M. Lotkin \& M. E. Young, Table of Binomial Coefficients. Exact Values. Ballistic Research Laboratories Memo. Report No. 762 Aberdeen Proving Ground, 1954, 49 p., $21.6 \times 27.9 \mathrm{~cm}$. Mimeographed from typescript.

This table is a sequel to RMT 1123. It gives exact values of the coefficients

$$
\frac{n !}{r !(n-r) !}
$$

for $r \leq(n+1) / 2$ and $n=0(1) 100$, whereas the previous table rounds these values to 20 figures. This new table will be of use in studies involving congruence properties and other theoretical properties of binomial coefficients.

D. H. L.

1235[C,D,E].-R. A. Hirvonen, "Nutshell tables of mathematical functions for interpolation with calculating machines," Bull. Géod. No. 30, 1953, p. 369-392.

The tables presented here are one page tables. Interpolation is by means of Taylor's development which the author writes in the nested form

$$
f(a+t h)=\{[(D t+C) t+B] t+A\} t+f(a)
$$

so that

$$
A=h f^{\prime}(a), \quad B=h^{2} f^{\prime \prime}(a) / 2 !, \ldots
$$

The tables therefore give the functions at coarse argument intervals together with the coefficients $A, B, C, D$. The tables are as follows:

$$
\begin{aligned}
\ln x \text { for } x & =1(.02) 1.6 ; 10 \mathrm{D} \\
e^{x} \text { for } x & =0(.01) .2 ; 10 \mathrm{D} \\
\sin x \text { for } x & =0^{\circ}\left(2^{\circ}\right) 90^{\circ} ; 10 \mathrm{D} \\
\arctan x \text { for } x & =0(.02) 1 ; 8 \mathrm{D} .
\end{aligned}
$$

